\title{
Adaptive Opportunistic Transmission in MU-MIMO Downlink with Reduced Feedback
}

\author{
Zhao Li, Jiawei Yang, Junliang Yao \\ State Key Laboratory of Integrated Service Networks, Xidian University, Xi' an 710071, China \\ Email: \{zli, jwyang\}@xidian.edu.cn, jlyao@pcn.xidian.edu.cn
}

\begin{abstract}
In this paper we proposed two threshold-aided adaptive opportunistic transmission strategies in multiuser multiple-input multiple-output (MU-MIMO) downlink with limited feedback. Threshold scheme in company with transmission mode adaptation are employed to handle feedback cost and improve system performance. In one time slot, each mobile station (MS) carries out channel estimation, selects appropriate transmission mode and feeds back adaptively. With feedback information the base station (BS) schedules one user out of candidate MSs and transmits to it. One of the proposed strategies employs single-level threshold. However, proper design of the threshold is difficult. Since a tight one would result in high outage probability whereas a loose one could not reduce feedback load effectively. Thus the other method employs double-level threshold. In this scheme, the primary threshold is used for feedback reduction, the secondary one is employed to guarantee the outage performance. In evaluating the strategies, statistical analysis and Monte-Carlo simulation are used. Results show that with properly designed thresholds, the proposed schemes can greatly reduce the feedback load, achieve high throughput and good outage performance.
\end{abstract}

Index Terms-Multiuser, MIMO, Downlink, Adaptive, Opportunistic, Threshold

\section{INTRODUCTION}

MU-MIMO is a set of advanced MIMO technologies that exploit the availability of multiple independent mobile users in order to improve communication performance. When multiple users are simultaneously supported by BS two major issues are under investigation, multiuser interference (MUI) elimination (precoding method design) [1-3] and multiuser scheduling [4-7]. These two issues are always discussed jointly and need channel state information (CSI) feedback. In existing MU-MIMO works, MUI elimination always induces much feedback load and computational complexity.

In a multiuser environment with multiple independent wireless links, at any given point in time the probability that all of those links have low quality is small. This advantage is called multiuser diversity (MUD). Accordingly, some works focus on opportunistic beamforming (OB) [813], i.e. in each time slot only one user among multiple candidates is selected to exploit MUD. Moreover, when $\mathrm{OB}$ is under discussion MUI is no longer considered.

Obviously, all of the above techniques require BS to know the channel information of users, which is usually

Manuscript received January 20, 2010; revised April 6, 2010; accepted May 12, 2010. estimated at the MS and fed to BS through a finite-rate reverse link. Since feedback information is part of system overhead, a lot of works focus on feedback load reduction. Most of them employ threshold to eliminate MSs whose channel quality is below the given threshold [6-9, 12-13], i.e. users who are not eligible for transmission would keep silent. However, these works are usually discussed in a simplified system model, i.e. the receiver end is equipped with single antenna $[8-9,12]$ or only part of multiple antennas is utilized [6]. As a result the advantages of MIMO are not fully exploited. Moreover, those works always consider fixed transmission mode. If adaptive transmission [14] is employed, not only the communication performance can be improved, the system feedback cost could also dynamically vary along with channel status. In [13] both threshold scheme and transmission mode adaptation are adopted. However, threshold design and mode selection criterion are not elaborated. Moreover, better performance may be achieved by modifying the number and redesigning the value of thresholds.

In this paper we proposed two adaptive opportunistic transmission strategies in MU-MIMO downlink. General MIMO scenario is considered in which both BS and MS are equipped with multiple antennas. Threshold scheme in company with transmission mode adaptation are employed to handle feedback cost and improve system performance. In one time slot, each MS carries out transmission mode selection and adaptively feeds CSI. With feedback information, BS schedules one user out of multiple candidates and implements transmission.

Among the proposed strategies, the first one employs single-level threshold, and the criterion of transmission mode adaptation is deduced from this threshold. However, proper design of the threshold is difficult, since a tight one would result in high outage probability whereas a loose one could not reduce feedback load effectively. Thus a double-level threshold scheme is introduced subsequently. In this strategy two thresholds are employed. The primary one is used for feedback reduction, the secondary one is to guarantee low outage probability.

The rest of this paper is organized as follows. In Section II we describe the system model. In Section III the single-level threshold strategy is introduced. Section IV presents the double-level threshold scheme. In the above two sections we also give the design of threshold values and analyzed the system performance theoretically. Section $\mathrm{V}$ provides the simulation results. Finally, the 
conclusion and future work are discussed in Section VI.

\section{System Model}

Consider a single cell MU-MIMO system with one BS and $K$ MSs. BS has $N_{T}$ transmit antennas and user- $k$ is equipped with $N_{k}$ receive antennas. Assume each MS undergoes statistically the same independent frequency non-selective fading. The channel matrix of user- $k$ in time slot $n$ is denoted by an $N_{k} \times N_{T}$ matrix $\mathbf{H}_{k}(n)$. Consider the Rayleigh block fading channel model. Make reasonable assumption that $N_{T}>N_{k}$, as MS always has rigorous size constraint compared with BS.

In a transmission cycle, BS should send training sequence to MSs, finish CSI collection and user selection before data transmission. These processes take a finite period of time called overhead slot. In downlink discussion we consider training sending as the primary overhead. Define $T$ and $\tau$ as the length of the total downlink time slot and overhead slot respectively. Similar to [15-16], typical values of $\tau / T$ lie in the region of $5 \%-10 \%$. For each user a low-rate error-free channel exists that conveys CSI back to the BS.

As we know, MIMO channel can be equivalent to a set of decoupled parallel subchannels by singular value decomposition (SVD). In slot $n$, assume that BS transmits to user- $k$ and the number of substreams for user- $k$ is $T_{k}(n)$, called mode, which satisfies $0 \leq T_{k}(n) \leq N_{k}$. When $T_{k}(n)=1$, BS transmits to MS- $k$ using beamforming $(\mathrm{BF})$. When $T_{k}(n)>1$, BS selects spatial multiplexing (SM). We employ a length- $T_{k}(n)$ vector $\mathbf{s}_{k}(n)$ to represent the data streams intended to user- $k$, satisfying $\varepsilon\left\{\left|s_{k, i}(n)\right|^{2}\right\}=1$.

Apply SVD to $\mathbf{H}_{k}(n)$, we have $\mathbf{H}_{k}(n)=$ $\mathbf{U}_{k}(n) \boldsymbol{\Lambda}_{k}(n) \mathbf{V}_{k}^{H}(n) . \mathbf{V}_{k}(n)$ is channel directional information (CDI) and $\boldsymbol{\Lambda}_{k}(n)$ is channel quality information (CQI) [17]. In this paper, we employ $\mathbf{V}_{k}(n)$ as the precoding matrix for user- $k . \mathbf{s}_{k}(n)$ is first transformed into a length- $N_{T}$ symbol vector by multiplying $\mathbf{V}_{k}(n)$. Then the symbol vector is launched from transmit antenna array. The power allocation matrix at $\mathrm{BS}$ is denoted as a $T_{k}(n) \times T_{k}(n)$ diagonal matrix $\mathbf{Q}_{k}(n)=\operatorname{diag}\left(q_{k, 1}(n), \cdots, q_{k, T_{k} n}(n)\right)$. The power assigned to user- $k$ satisfies total transmit power constraint $P_{T}=\operatorname{tr}\left(\mathbf{Q}_{k}(n)\right)=\sum_{i=1}^{T_{k}(n)} q_{k, i}(n)$.

At the user side, the received signal vector for user- $k$ is

$$
\mathbf{r}_{k}(n)=\mathbf{H}_{k}(n) \mathbf{V}_{k}(n) \mathbf{Q}_{k}^{1 / 2}(n) \mathbf{s}_{k}(n)+\mathbf{n}_{k}(n)
$$

where $\mathbf{Q}_{k}^{1 / 2}(n)$ stands for the algebra square root operation of $\mathbf{Q}_{k}(n)$ 's entries. $\mathbf{n}_{k}(n)$ is the noise vector whose elements are i.i.d. zero mean complex Gaussian random variable with variance $N_{0}$.

User- $k$ generates an estimate $\overline{\mathbf{r}}_{k}(n)$ for $\mathbf{r}_{k}(n)$ by multiplying the conjugate transpose of left singular vector matrix $\mathbf{U}_{k}(n)$,

$$
\overline{\mathbf{r}}_{k}(n)=\boldsymbol{\Lambda}_{k}(n) \mathbf{Q}_{k}^{1 / 2}(n) \mathbf{s}_{k}(n)+\mathbf{U}_{k}^{H}(n) \mathbf{n}_{k}(n)
$$

Water-flling power allocation [18] could achieve the optimal throughput performance. However, in many practical systems it puts a high demand on the linear range of transmit power amplifiers, which is extremely costly especially for multiple antenna systems. Thus in this paper, equal power allocation [14, 19] is used at BS. Moreover, we assume each MS has 2 antennas. The time index $n$ is omitted for simplicity when discussing within a single time slot.

\section{Single-Level Threshold AdAPtive TRANSMISSION STRATEGY}

\section{A. Feedback strategy at MS}

Recall that $N_{T}>N_{k}$ and each MS is equipped with 2 antennas, i.e. $\operatorname{rank}\left(\mathbf{H}_{k}\right)=2$ and $T_{k} \in\{1,2\}$. Use $R_{k}^{B F}$ and $R_{k}^{S M}$ to denote the achievable rate of user- $k$ in one time slot using BF and SM, respectively. They are given by (3) and (4), where $B$ denotes the transmission bandwidth.

$$
\begin{gathered}
R_{k}^{B F}=B \log _{2}\left(1+P_{T} \lambda_{k, 1}^{2} / N_{0}\right) \\
R_{k}^{S M}=B \sum_{i=1}^{T_{k}} \log _{2}\left(1+\frac{P_{T} \lambda_{k, i}^{2}}{N_{T} N_{0}}\right)
\end{gathered}
$$

$\lambda_{k, 1}$ and $\lambda_{k, i}$ stand for the maximum and the $i$ th singular value of $\mathbf{H}_{k}$, respectively. If SM achieves higher capacity multiple subchannels are activated for transmission, otherwise BF is better and only the principal eigenmode is selected.

We employ single-level threshold $Q_{p r i}^{t h}$ in the strategy. In order to implement adaptive transmission we introduce another threshold $Q_{\text {sec }}^{t h}$ derived from $Q_{\text {pri }}^{t h}$, which will be discussed later. In one slot $n$, MSs decide locally whether they should attempt to access the channel and send feedback to the BS or not. In the negative case, they remain silent for that slot. The feedback strategy at MS is described in Fig. 1, where $\mathbf{v}_{k, 1}$ is the principal right singular vector (PRSV) corresponding to $\lambda_{k, 1}$.

\section{B. Scheduling strategy at BS}

On receiving feedback information, BS selects one user from the pool of eligible MSs [8]. We assume both CDI and CQI are available at BS and employ greedy scheduling strategy as described in Fig. 2, where $L$ denotes the number of eligible users in one slot.

It is possible that for some time slots the BS cannot identify any eligible users to be considered for transmission. This happens in the case where no user has good enough channel gain higher than the preset threshold. Thus the number of feedback users is zero. This condition is defined as outage. In our discussion BS terminates transmission when outage occurs, i.e. the throughput of an outage slot is 0 . 


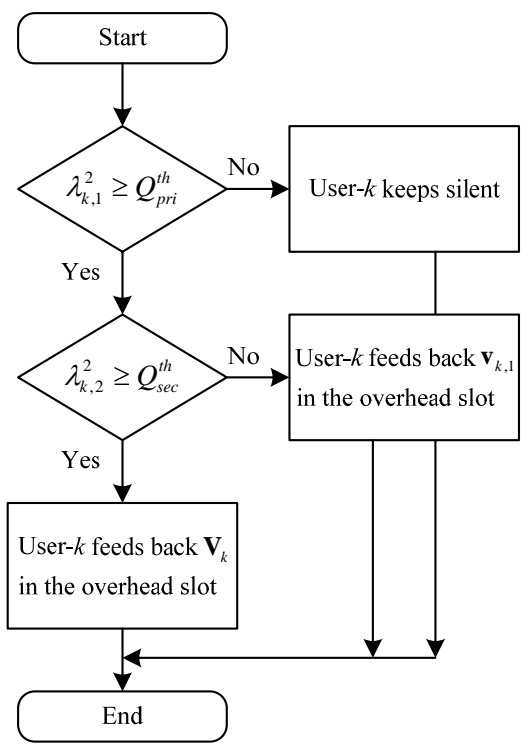

Figure 1. Feedback strategy at MS applying single-level threshold.

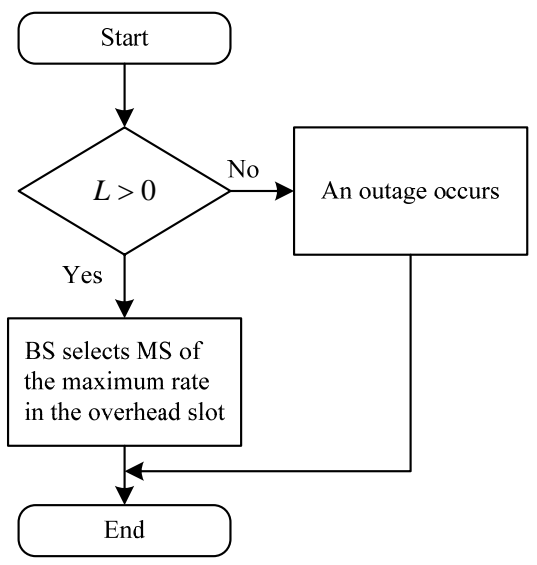

Figure 2. Scheduling strategy at BS applying single-level threshold.

\section{Threshold design}

Most existing works discussed the threshold design under simplified system model and resulted in the closedform formulas [20]. As in general MIMO scenario the distribution of $\lambda_{k, i}^{2}$ is too complicated, we use numerical searching method to find $Q_{p r i}^{t h}$.

We assume each right singular vector (RSV) $\mathbf{v}_{k, i}$ is quantized into $M$ bits, and antenna configuration is $N_{T}=8, N_{k}=2$. Fig. 3 and Fig. 4 illustrate the throughput (normalized by $B$ and $T$ ) and the average CDI feedback load (normalized by $M$ ) under $\tau / T=5 \%$, $K=10$, different $Q_{p r i}^{t h}$ and SNR (Signal to noise ratio), respectively.

From Fig. 3 we can see that as $Q_{p r i}^{t h}$ becomes larger than $10.5 \mathrm{~dB}$ the throughput starts to decrease notably. In Fig. 4 the feedback load monotonically reduces with increasing $Q_{p r i}^{t h}$. Based on both figures it can be concluded that under $K=10$, in order to maintain good system throughput performance as well as low feedback load, the appropriate value of primary threshold should be $10.5 \mathrm{~dB}$. The same method can be applied to determine $Q_{p r i}^{t h}$ when

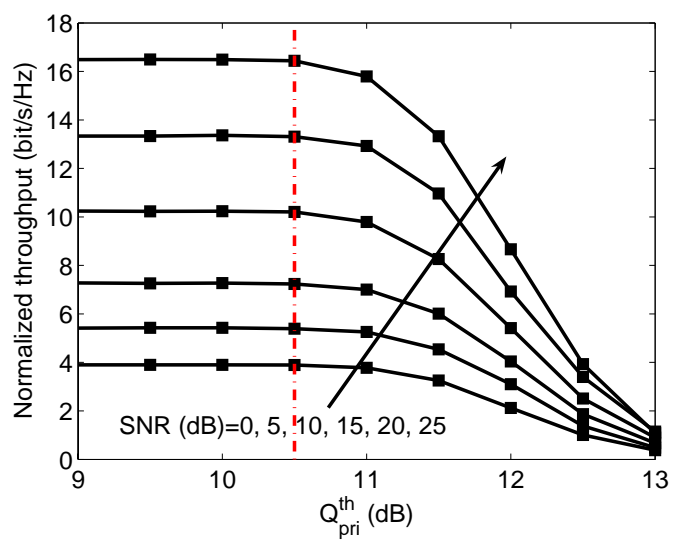

Figure 3. Normalized throughput per time slot under $\tau / T=5 \%$, $K=10$, different $Q_{p r i}^{t h}$ and SNR.

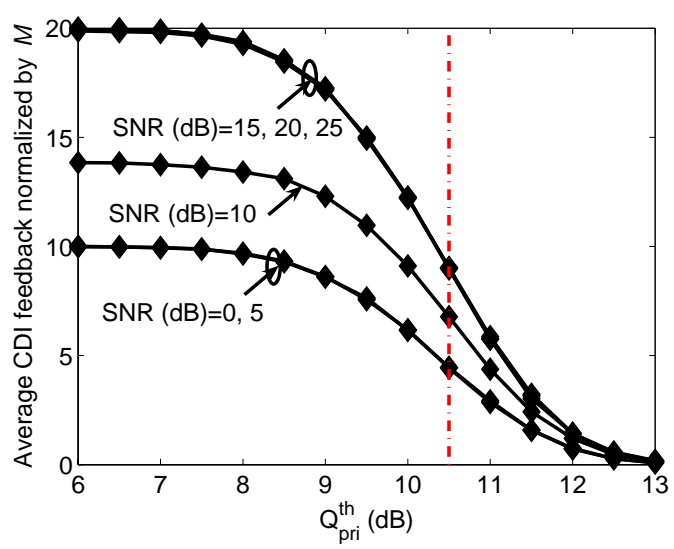

Figure 4. Average CDI feedback load per time slot normalized by $M$ under $K=10$, different $Q_{p r i}^{t h}$ and SNR.

the number of users is taken other values. $Q_{p r i}^{t h}$ under different $K$ are presented in Table I.

In practical the threshold level can be set by the BS based on CSI collection within a certain period of time. Moreover, the threshold value could be adaptive to the communication environment to achieve better performance.

TABLE I.

$Q_{p r i}^{t h}$ Values Applying Single-LeVel Threshold

\begin{tabular}{|l|l|l|l|l|l|l|l|}
\hline$K$ & 3 & 5 & 10 & 15 & 20 & 30 & 40 \\
\hline$Q_{\text {pri }}^{t h}(\mathrm{~dB})$ & 9.5 & 10.0 & 10.5 & 10.8 & 11.0 & 11.3 & 11.5 \\
\hline
\end{tabular}

According to the algorithm in Fig. 1, we design $Q_{\text {sec }}^{\text {th }}$ as follows. From (3) and (4) we have

$$
R_{k}^{S M} \geq R_{k}^{B F} \Leftrightarrow \lambda_{k, 2}^{2} \geq \frac{\left(N_{T}-1\right) \lambda_{k, 1}^{2}}{1+\gamma_{0} \lambda_{k, 1}^{2} / N_{T}}
$$

where $\gamma_{0}=P_{T} / N_{0}$. The realtionship of $Q_{p r i}^{t h}$ and $Q_{s e c}^{t h}$ is given by (6),

$$
Q_{\text {sec }}^{t h}=\frac{\left(N_{T}-1\right) Q_{\text {pri }}^{t h}}{1+\gamma_{0} Q_{\text {pri }}^{t h} / N_{T}}+\beta
$$


$\beta$ is the function of $\lambda_{k, 1}$ and $Q_{p r i}^{t h}$,

$$
\beta=N_{T} \frac{\lambda_{k, 1}^{2}-Q_{p r i}^{t h}}{\left(1+\gamma_{0} Q_{p r i}^{t h} / N_{T}\right)^{2}}
$$

The derivation of $\beta$ is described in Appendix. $Q_{\text {sec }}^{\text {th }}$ for each time slot could be readily obtained by using (6), (7) and values in Table I. Moreover, from (7) it can be seen that with fixed $Q_{p r i}^{t h}$, larger $\lambda_{k, 1}$ would result in greater $\beta$ as well as $Q_{\text {sec }}^{t h}$.

\section{Performance analysis}

In this section we give the theoretical analysis of downlink throughput and system feedback load. Recall that $L$ denotes the number of eligible MSs in one slot. According to the algorithm in Fig. 1, the throughput normalized by $B$ and $T$ is as follows,

$$
T h=\left\{\begin{array}{cr}
\left(1-\frac{\tau}{T}\right) \max _{k=1, \cdots, L}\left(R_{k}^{B F}, R_{k}^{S M}\right), & L>0 \\
0, & \text { otherwise }
\end{array}\right.
$$

The CDI feedback load (normalized by $M$ ) of $L$ eligible users of which $l$ users select BF in one slot, is given by (9).

$$
F(L, l)=\left\{\begin{array}{cc}
2 M L-M l, & L>0 \\
0, & \text { otherwise }
\end{array}\right.
$$

Since one RSV corresponds to one singular value, CDI load varies in the same manner as CQI load does. Thus the later is not investigated in this paper.

Define the cumulative distribution function (CDF) of the $i$ th eigenvalue $\zeta_{k, i}$ of $\mathbf{H}_{k} \mathbf{H}_{k}^{H}$ as $P_{\zeta_{k, i}}(u), i \in\{1,2\}$. And $p_{\zeta_{k, i}}(u)$ represents the corresponding probability density function (PDF). For example, the probability that $\zeta_{k, i}$ is less than $Q_{p r i}^{t h}$ is $P_{\zeta_{k, i}}\left(Q_{p r i}^{t h}\right)$. Note that $\zeta_{k, i}=\lambda_{k, i}^{2}$. Based on the assumption that each user undergoes statistically the same independent fading, for any $k \in\{1, \cdots, K\}$ we have $P_{\zeta_{k, i}}(u)=P_{\zeta_{i}}(u)$ and $p_{\zeta_{k, i}}(u)=p_{\zeta_{i}}(u)$.

The probabilities that an arbitrary user is eligible for feeding information in an overhead slot is

$$
P^{f d}=1-P_{\zeta_{1}}\left(Q_{\text {pri }}^{t h}\right)
$$

In Table II the possible states of MS are listed.

TABLE II.

Possible States of MS ApPlying Single-Level Threshold

\begin{tabular}{|l|l|}
\hline State & Description \\
\hline$S^{B F}$ & Select BF and feed $\mathbf{v}_{k, 1}$ in the overhead slot. \\
\hline$S^{S M}$ & Select SM and feed $\mathbf{V}_{k, 1}$ in the overhead slot. \\
\hline$S_{o}$ & MS stays quiet. \\
\hline
\end{tabular}

(11)-(13) give the probabilities that an arbitrary MS is in one of the above states, respectively.

$$
\begin{gathered}
P^{B F}=\operatorname{Prob}\left(S^{B F}\right)=P^{f d} P_{\zeta_{2}}\left(Q_{\text {sec }}^{t h}\right) \\
P^{S M}=\operatorname{Prob}\left(S^{S M}\right)=P^{f d}\left(1-P_{\zeta_{2}}\left(Q_{\text {sec }}^{t h}\right)\right) \\
P_{o}=\operatorname{Prob}\left(S_{o}\right)=1-P^{f d}
\end{gathered}
$$

From (11)-(13) we can easily have

$$
P^{B F}+P^{S M}+P_{o}=1
$$

Denote the number of eligible users in one slot as $\mathscr{N}$. We have

$$
\begin{gathered}
P_{L}=\operatorname{Prob}(\mathscr{N}=L)=\left(\begin{array}{c}
K \\
L
\end{array}\right)\left(P^{f d}\right)^{L}\left(1-P^{f d}\right)^{K-L} \\
P_{o}^{\text {sys }}=\operatorname{Prob}(\mathscr{N}=0)=\left(1-P^{f d}\right)^{K}
\end{gathered}
$$

(15) denotes the probability that the number of eligible MSs in one slot is $L$. (16) indicates the system outage probability.

From (10)-(16), the expectation of throughput and CDI feedback load can be readily obtained,

$$
\mathbb{E}(T h)=\left(1-\left(P_{\zeta_{1}}\left(Q_{\text {pri }}^{t h}\right)\right)^{K}\right)\left(1-\frac{\tau}{T}\right) E(\Phi)
$$

where

$$
\Phi=\max _{k=1, \cdots, L}\left(R_{k}^{B F}, R_{k}^{S M}\right)
$$

Note that $Q_{\text {sec }}^{t h}$ is function of $\lambda_{k, 1}$, which is a random variable. In statistical analysis we use the expectation $\mathbb{E}\left(Q_{\text {sec }}^{t h}\right)$ instead of $Q_{s e c}^{t h}$ to compute (11) and (12).

In order to calculate (10)-(18), the PDF of $\zeta_{k, i}$ should be obtained beforehand. This distribution is given in [20] as follows,

$$
p_{\zeta_{(\cdot)}}(u)=\varphi \sum_{n=1}^{N_{k}} \sum_{m=1}^{N_{k}}(-1)^{n+m} u^{\alpha} \exp ^{-u}\left|\boldsymbol{\Omega}_{(\cdot)}\right|
$$

where $\alpha=n+m-2+N_{T}-N_{k}$. In this paper $p_{\zeta_{(\cdot)}} \in$ $\left\{\zeta_{1}, \zeta_{2}\right\}, \zeta_{1}>\zeta_{2} \cdot \varphi$ is a constant. $\boldsymbol{\Omega}_{(\cdot)}$ is an $\left(N_{k}-1\right) \times$ $\left(N_{k}-1\right)$ square matrix. For room limitation the details of (20) are not elaborated.

\section{Double-Level Threshold Adaptive TRANSMISSION STRATEGY}

As for the single-level threshold transmission strategy, proper design of the threshold is difficult. Since a tight one would result in high outage probability whereas a loose one could not reduce feedback load effectively. Thus a double-level threshold scheme is proposed in this section.

\section{A. Feedback strategy at MS}

Similar to the algorithm in Fig. 1, we adopt two overhead slots here. In the first one, two tight thresholds $Q_{1, p r i}^{t h}$ and $Q_{1, \text { sec }}^{t h}$ are employed to implement user and transmission mode selection, respectively. In the second overhead slot, loose thresholds $Q_{2, p r i}^{t h}$ and $Q_{2, s e c}^{t h}$ are used. As depicted in Section III, $Q_{m, s e c}^{t h}$ is derived from $Q_{m, p r i}^{t h}$ $(m=1,2)$. In one slot $n$, the feedback algorithm at MS is given in Fig. 5.

\section{B. Scheduling strategy at BS}

The scheduling algorithm at BS is given in Fig. 6, where $L_{1}$ and $L_{2}$ denote the number of eligible users in the first and second overhead slot, respectively. We assume that BS terminates transmission when outage takes place. 


$$
\mathbb{E}(F)=\sum_{L=1}^{K}\left\{\left(\begin{array}{c}
K \\
L
\end{array}\right)\left(1-P^{f d}\right)^{K-L} \sum_{l=0}^{L}\left[\left(\begin{array}{c}
L \\
l
\end{array}\right)\left(P^{B F}\right)^{l}\left(P^{S M}\right)^{L-l}(2 M L-M l)\right]\right\}
$$

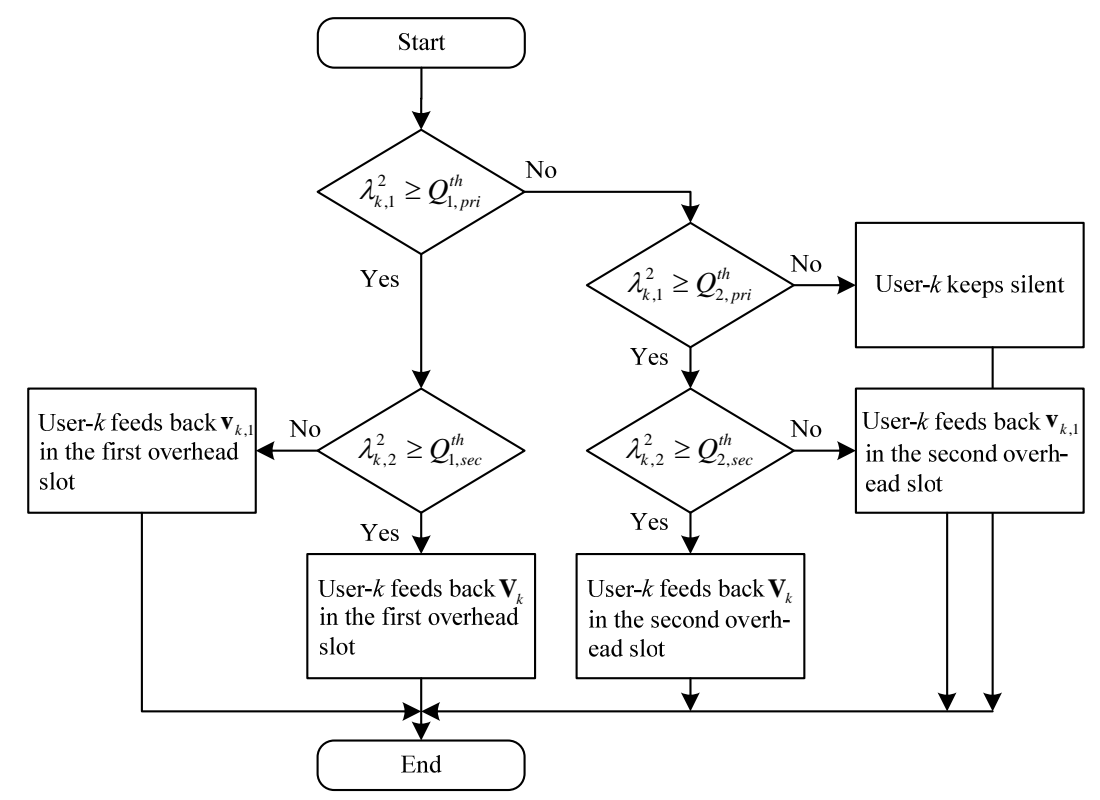

Figure 5. Feedback strategy at MS applying double-level threshold.

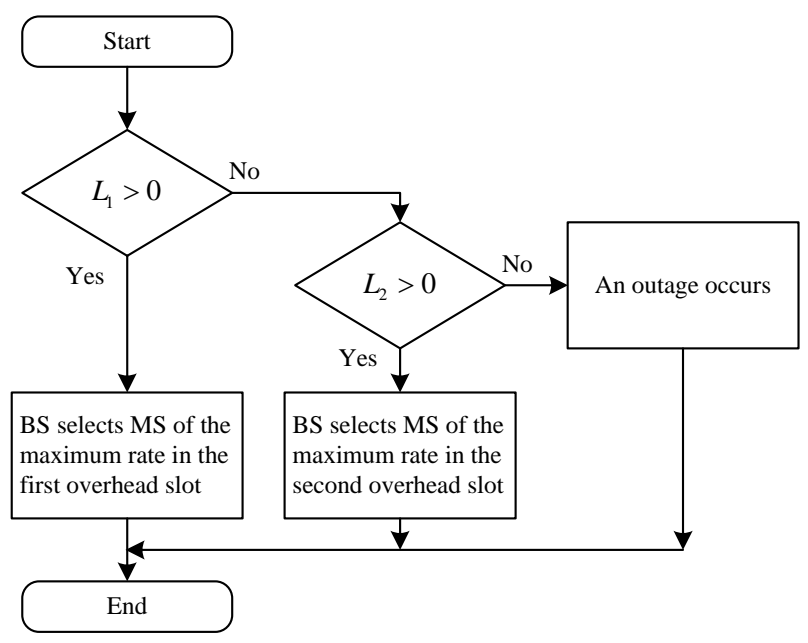

Figure 6. Scheduling strategy at BS applying double-level threshold.

\section{Threshold design}

We adopt the same idea as described in Section III. Accordingly, (6) and (7) can be rewritten in general form as follows,

$$
Q_{m, s e c}^{t h}=\frac{\left(N_{T}-1\right) Q_{m, p r i}^{t h}}{1+\gamma_{0} Q_{m, p r i}^{t h} / N_{T}}+\beta_{m}
$$

where $m=1,2 . \beta_{m}$ is given by (22),

$$
\beta_{m}=N_{T} \frac{\lambda_{k, 1}^{2}-Q_{m, p r i}^{t h}}{\left(1+\gamma_{0} Q_{m, p r i}^{t h} / N_{T}\right)^{2}}
$$

Recall that $Q_{2, p r i}^{t h}$ is to guarantee the system outage performance. In order to make comparison to the single- level threshold strategy, $Q_{2, p r i}^{t h}$ is designed following the results in Table I. As a result the outage probabilities of both strategies would be the same.

Note that $Q_{1, p r i}^{t h}$ should be designed to effectively reduce the number of feedback users in the first overhead slot, thus it should be greater than $Q_{2, p r i}^{t h}$. Define the gap between these two thresholds as $\delta=Q_{1, p r i}^{t h}-Q_{2, p r i}^{t h}$. Fig. 7 illustrates the normalized average CDI feedback load per time slot under $K=10$, different $\delta$ and SNR values.

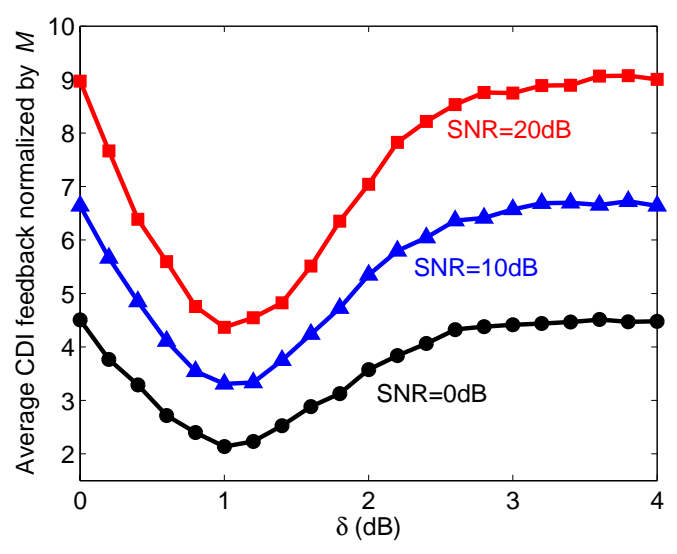

Figure 7. Average CDI feedback load per time slot normalized by $M$ under $K=10$, different $\delta$ and SNR.

As shown in the figure, when $\delta=0 \mathrm{~dB}$ the feedback load of proposed scheme is the same with that of the single-level one, which can also be referred to Fig. 11. With increasing $\delta, Q_{1, \text { pri }}^{\text {th }}$ becomes aggressive and results in decreasing number of eligible users. When $\delta<1 \mathrm{~dB}$, 


$$
\begin{aligned}
& T h=\left\{\begin{array}{cc}
\left(1-\frac{\tau}{T}\right) \max _{k=1, \cdots, L_{1}}\left(R_{k}^{B F}, R_{k}^{S M}\right), & L_{1}>0 \\
\left(1-2 \frac{\tau}{T}\right) \max _{k=1, \cdots, L_{2}}\left(R_{k}^{B F}, R_{k}^{S M}\right), & L_{1}=0 \quad \text { and } \quad L_{2}>0 \\
0, & \text { otherwise }
\end{array}\right. \\
& P_{o}=\operatorname{Prob}\left(S_{o}\right)=P_{\zeta_{1}}\left(Q_{1, p r i}^{t h}\right)\left(1-\left(P_{\zeta_{1}}\left(Q_{1, p r i}^{t h}\right)\right)^{K-1}\right)+P_{\zeta_{1}}\left(Q_{2, p r i}^{t h}\right)\left(P_{\zeta_{1}}\left(Q_{1, p r i}^{t h}\right)\right)^{K-1} \\
& \mathbb{E}(T h)=\left(1-\left(P_{\zeta_{1}}\left(Q_{1, p r i}^{t h}\right)\right)^{K}\right)\left(1-\frac{\tau}{T}\right) E\left(\Phi_{1}\right)+\left(P_{\zeta_{1}}\left(Q_{1, p r i}^{t h}\right)\right)^{K}\left(1-\left(1-P_{2}^{f d}\right)^{K}\right)\left(1-2 \frac{\tau}{T}\right) E\left(\Phi_{2}\right) \\
& \mathbb{E}(F)=\sum_{m=1}^{2} \sum_{L_{m}=1}^{K}\left(\begin{array}{c}
K \\
L_{m}
\end{array}\right)\left(1-P_{m}^{f d}\right)^{K-L_{m}} \sum_{l_{m}=0}^{L_{m}}\left[\left(\begin{array}{c}
L_{m} \\
l_{m}
\end{array}\right)\left(P_{m}^{B F}\right)^{l_{m}}\left(P_{m}^{S M}\right)^{L_{m}-l_{m}}\left(2 M L_{m}-M l_{m}\right)\right]
\end{aligned}
$$

although the outage probability increases, the probability that scheduling can be carried out in the first overhead slot is still high. Thus the feedback load reduces along with increasing $\delta$ (under $\delta<1 \mathrm{~dB}$ ). When $\delta$ grows larger than $1 \mathrm{~dB}$, the probability that the second overhead slot is needed for scheduling becomes higher, consequently the feedback load (yielded in two overhead slots) starts to increase. As $\delta$ becomes too large, the outage probability in the first overhead slot approximates to 1 and both schemes result in the same cost. Based on the discussion above, there exists an optimal $\delta$ to minimize the feedback load. As shown in Fig. 7 the proper $\delta$ is $1 \mathrm{~dB}$ under $K=10$.

We use numerical searching method to find $Q_{1, \text { pri }}^{t h}$, the results are given in Table III.

TABLE III.

$Q_{1, p r i}^{t h}$ Values Applying Double-Level Threshold

\begin{tabular}{|l|l|l|l|l|l|l|l|}
\hline$K$ & 3 & 5 & 10 & 15 & 20 & 30 & 40 \\
\hline$Q_{1, p r i}^{t h}(\mathrm{~dB})$ & 10.8 & 11.1 & 11.5 & 11.7 & 11.9 & 12.1 & 12.3 \\
\hline
\end{tabular}

\section{Performance analysis}

In this section we give the theoretical analysis applying double-threshold scheduling strategy. Denote $L_{1}$ and $L_{2}$ as the number of eligible MSs in the first and second overhead slot, respectively. The throughput normalized by $B$ and $T$ is given by (23).

Note that when $L_{1}=0$ and $L_{2}>0$, due to an outage occurs in the first overhead slot the throughput of doublelevel threshold scheme is inferior to that of the single-level one, i.e. throughput loss is resulted.

The normalized CDI feedback load of $L_{m}$ eligible users, of which $l_{m}$ users select BF in the $m$ th $(m=1,2)$ overhead slot is

$F\left(L_{m}, l_{m}\right)=\left\{\begin{array}{cc}2 M L_{1}-M l_{1}, & L_{1}>0 \\ 2 M L_{2}-M l_{2}, & L_{1}=0 \text { and } L_{2}>0 \\ 0, & \text { otherwise }\end{array}\right.$

The probabilities that an arbitrary user is eligible for feeding information in the first and second overhead slot are

$$
P_{1}^{f d}=1-P_{\zeta_{1}}\left(Q_{1, p r i}^{t h}\right)
$$

$$
P_{2}^{f d}=\left(1-P_{1}^{f d}\right)^{K-1}\left(P_{\zeta_{1}}\left(Q_{1, p r i}^{t h}\right)-P_{\zeta_{1}}\left(Q_{2, p r i}^{t h}\right)\right)
$$

Each MS has five states, listed in Table IV.

TABLE IV.

Possible States of MS APPlying Double-LeVEl Threshold

\begin{tabular}{|l|l|}
\hline State & Description \\
\hline$S_{1}^{B F}$ & Select BF and feed $\mathbf{v}_{k, 1}$ in the overhead slot. \\
\hline$S_{1}^{S M}$ & Select SM and feed $\mathbf{V}_{k, 1}$ in the overhead slot. \\
\hline$S_{2}^{B F}$ & $\begin{array}{l}\text { Outage occurs in the first overhead slot. } \\
\text { Select BF and feed } \mathbf{v}_{k, 1} \text { in the second overhead slot. }\end{array}$ \\
\hline$S_{2}^{S M}$ & $\begin{array}{l}\text { Outage occurs in the first overhead slot. } \\
\text { Select } \mathrm{SM} \text { and feed } \mathbf{V}_{k, 1} \text { in the second overhead slot. }\end{array}$ \\
\hline$S_{o}$ & MS stays quiet. \\
\hline
\end{tabular}

(27)-(29) give the probabilities that an arbitrary MS is in one of the above states.

$$
\begin{gathered}
P_{m}^{B F}=\operatorname{Prob}\left(S_{m}^{B F}\right)=P_{m}^{f d} P_{\zeta_{2}}\left(Q_{m, s e c}^{t h}\right) \\
P_{m}^{S M}=\operatorname{Prob}\left(S_{m}^{S M}\right)=P_{m}^{f d}\left(1-P_{\zeta_{2}}\left(Q_{m, s e c}^{t h}\right)\right)
\end{gathered}
$$

The first part in the RHS (Right hand side) of (29) denotes the probability that an arbitrary MS is ineligible in the first overhead slot and at least one of the other $K-1$ users is eligible for transmission. The second part indicates the probability that both the MS in the second overhead slot and the other $K-1$ users in the first overhead slot are ineligible for transmission.

From (27)-(29) we can easily have

$$
\sum_{m=1}^{2}\left(P_{m}^{B F}+P_{m}^{S M}\right)+P_{o}=1
$$

Denote the number of eligible users in the $m$ th overhead slot for an arbitrary slot as $\mathscr{N}_{m}(m=1,2)$. We have

$$
\begin{aligned}
P_{L_{m}} & =\operatorname{Prob}\left(\mathscr{N}_{m}=L_{m}\right) \\
& =\left(\begin{array}{c}
K \\
L_{m}
\end{array}\right)\left(P_{m}^{f d}\right)^{L_{m}}\left(1-P_{m}^{f d}\right)^{K-L_{m}}
\end{aligned}
$$

From (27)-(29) and (31), the expectation of throughput and CDI feedback load can be readily obtained in (32) and (33). $\Phi_{m}=\max _{k=1, \cdots, L_{m}}\left(R_{k}^{B F}, R_{k}^{S M}\right)$. Similar to the discussion in Section III, we also use $\mathbb{E}\left(Q_{m, s e c}^{t h}\right)$ instead of $Q_{m, s e c}^{t h}$ in theoretical analysis. 


\section{Simulation Results}

In this section, we use simulation results to illustrate the performance of the proposed scheduling strategies. Assume $K \in\{3,10,20,30,40\}$ and antenna configuration $N_{T}=8, N_{k}=2$. Six strategies are under investigation: $G S_{B F} w o T$ (Greedy scheduling of BF users without threshold), GS $S_{S M} w o T$ (Greedy scheduling of SM users without threshold), GS $S_{M A} w o T$ (Greedy scheduling of mode-adaptive users without threshold), GS $S_{M A} w S L T$ (Greedy scheduling of mode-adaptive users with singlelevel loose threshold, applying the values in Table I), $G S_{M A} w S T T$ (Greedy scheduling of mode-adaptive users with single-level tight threshold, applying the values in Table III) and $G S_{M A} w D T$ (Greedy scheduling of modeadaptive users with double-level threshold). The first two schemes are similar to the conventional OB [8]. For the strategies without threshold we also consider one overhead slot overhead for BS to send training sequence.

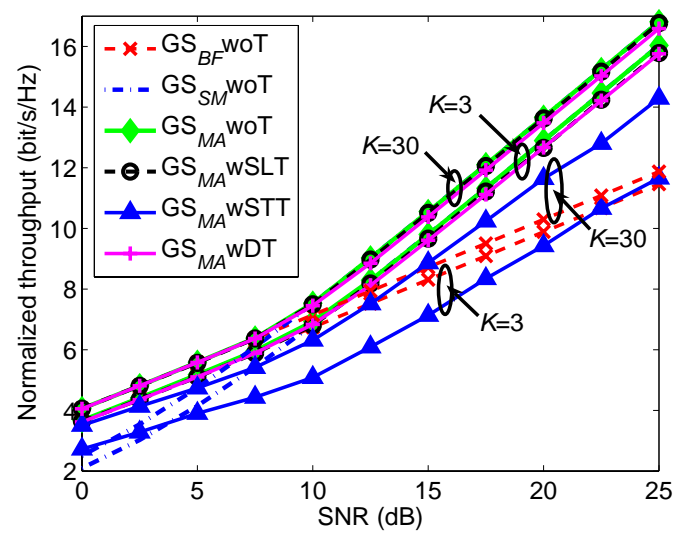

Figure 8. Normalized throughput per time slot with different strategies under $\tau / T=5 \%, K=3,30$ and different SNR.

Fig. 8 shows the normalized throughput per time slot for different schemes under $\tau / T=5 \%, K=3,30$ and different SNR. As shown in the figure, $G S_{B F} w o T$ performs better at low SNR whereas $G S_{S M} w o T$ achieves larger throughput at high SNR. Without threshold, every MS feeds CSI and the one of the maximum rate can be activated using greedy scheduling scheme. Thus $G S_{M A} w o T$ is throughput optimal. With threshold, only eligible users feed information. The outage probability of a small $K$ system is higher than that of a system with large $K$. Thus the throughputs of $G S_{M A} w S L T$, $G S_{M A} w S T T$ and $G S_{M A} w D T$ grow with increasing $K$. As figure shows, $G S_{M A} w S L T$ and $G S_{M A} w D T$ can achieve almost the same near-optimal throughput. This is because both schemes apply the same loose threshold values. Furthermore, since tight threshold results in high outage probability, the throughput of $G S_{M A} w S T T$ is poor compared with that of $G S_{M A} w S L T$ and $G S_{M A} w D T$.

From Fig. 8 it can be concluded that $G S_{M A} w S T T$ results in poor throughput performance. $G S_{M A} w S L T$ and $G S_{M A} w D T$ are of almost the same near-optimal throughput. As discussed in Section IV, double-level

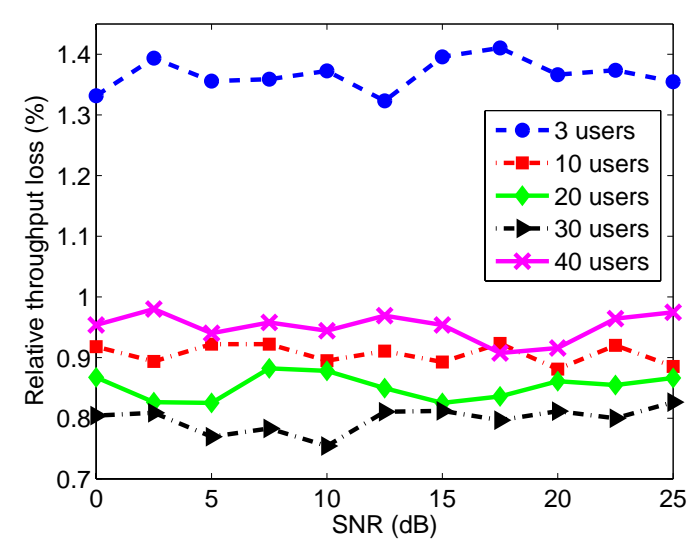

Figure 9. Relative throughput loss of $G S_{M A} w D T$ with respect to $G S_{M A} w S L T$ under $\tau / T=5 \%$, different $K$ and SNR.

threshold strategy may incur throughout loss with respect to the single-level one. In order to illustrate the difference between $G S_{M A} w S L T$ and $G S_{M A} w D T$, Fig. 9 shows the relative throughput loss $\left(R L_{T h}\right)$ of $G S_{M A} w D T$ with respect to $G S_{M A} w S L T$ under $\tau / T=5 \%$, different $K$ and SNR. $R L_{T h}$ is defined in (34),

$$
R L_{T h}=\frac{T h_{S L T}-T h_{D T}}{T h_{S L T}} \times 100 \%
$$

where $T h_{S L T}$ and $T h_{D T}$ denote the throughput of $G S_{M A} w S L T$ and $G S_{M A} w D T$, respectively. Note that with properly designed $Q_{1, p r i}^{t h}$, BS starts data transmission immediately following the first overhead slot with high probability, thus both strategies are of almost the same throughput performance. As shown in the figure, $R L_{T h}$ keeps low in the SNR region.

We may notice in Fig. 9 that the relative throughput loss for 40 users is less than for 3 users but greater than for 10, 20 and 30 users. This is due to the fact that the threshold values given in Table III for 40 users is a bit tighter, thus resulted in the higher probability that the second overhead slot is taken for scheduling which could have been used for data transmission. If the threshold is adjusted elaborately, the throughput loss would be reduced.

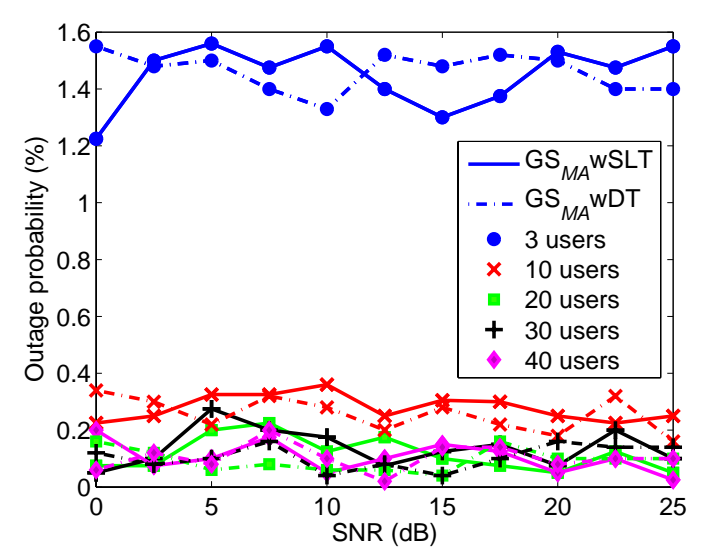

Figure 10. Outage probability of $G S_{M A} w S L T$ and $G S_{M A} w D T$ under different $K$ and SNR. 
Fig. 10 shows the outage probability of $G S_{M A} w S L T$ and $G S_{M A} w D T$ under different $K$ and SNR values. The outage probability of $G S_{M A} w S T T$ ranges from $14 \%$ to $28 \%$ when $K$ varies from 3 to 40 . For space limitation these curves of poor performance are not plotted. As shown in Fig. 10, both schemes produce low outage probability in the SNR region. Their outage performance is statistically the same, which is consistent with the analysis in Section IV. As $K$ increases, the outage probability approximates to zero. Since each MS undergoes independent fading, the probability that all users are in bad condition diminishes along with increasing $K$.

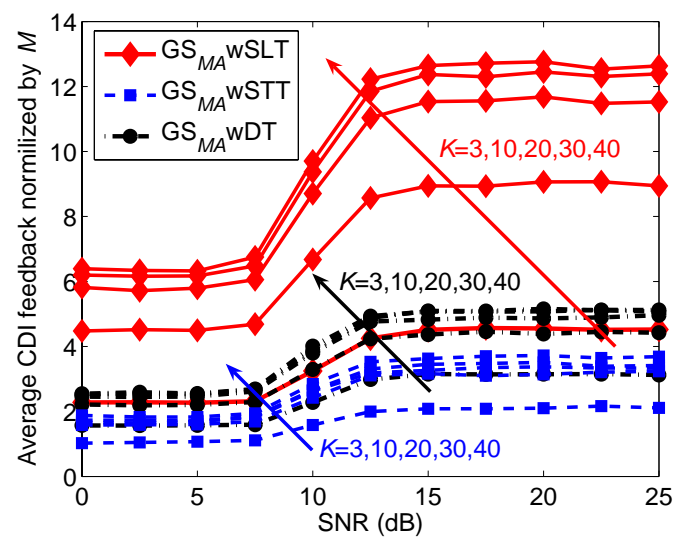

Figure 11. Average CDI feedback load per time slot normalized by $M$ under different $K$ and SNR.

Fig. 11 shows the average CDI feedback load per time slot normalized by $M$. The load of $G S_{B F} w o T$, $G S_{S M} w o T$ and $G S_{M A} w o T$ are equal to $K, 2 K$ and varies from $K$ to $2 K$ along with increasing SNR, respectively [13]. For clarity, these curves are not plotted. As shown in the figure, BF is preferable at low SNR. SM becomes better at higher SNR, accordingly the load increases. When SNR $>15 \mathrm{~dB}$, nearly all users select SM. With properly designed threshold, only limited number of eligible users feed information. Thus with fixed $K$ the load of $G S_{M A} w S T T, G S_{M A} w S L T$ and $G S_{M A} w D T$ converges to an asymptotic value as SNR increases. As can be seen from the figure, applying $G S_{M A} w D T$ the feedback load can be significantly reduced compared with $G S_{M A} w S L T$. The load of $G S_{M A} w S T T$ is comparable with that of $G S_{M A} w D T$, yet the former is of poor outage performance.

Fig. 12 and Fig. 13 show the Normalized throughput per time slot and average CDI feedback load normalized by $M$ using $G S_{M A} w D T$, respectively. Monte-Carlo simulation and statistical analysis are employed. It can be seen that the analytical results are consistent with the simulative one. When $G S_{M A} w S L T$ or $G S_{M A} w S T T$ is under investigation, the similar consistency can be obtained.

\section{CONCLUSION AND Future WORK}

In wireless communications especially in multiuser scenarios, achieving good system performance with re-

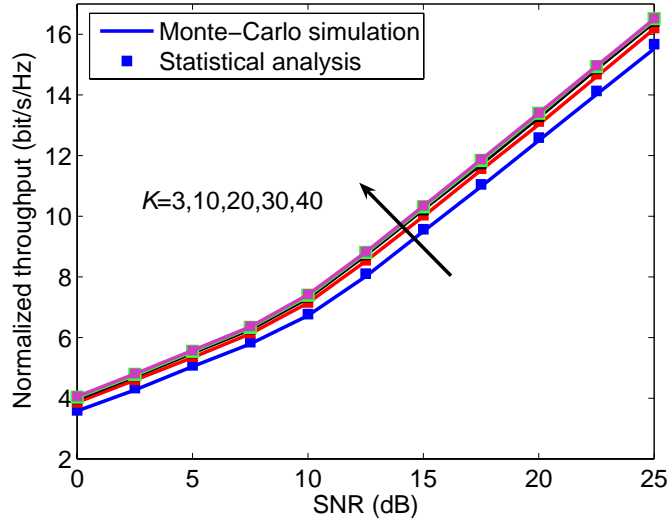

Figure 12. Normalized throughput per time slot under different $K$ and SNR using Monte-Carlo simulation and statistical analysis.

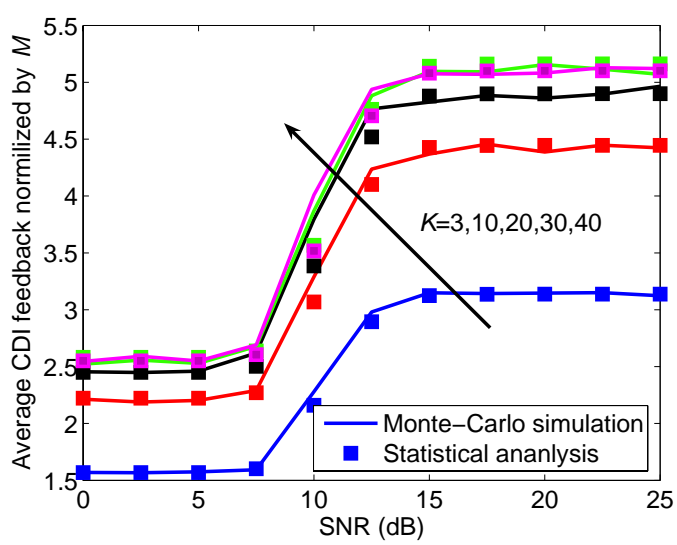

Figure 13. Average CDI feedback load per time slot normalized by $M$ under different $K$ and SNR using Monte-Carlo simulation and statistical analysis.

duced cost is an interesting research issue. In this paper we proposed two adaptive opportunistic transmission strategies in MU-MIMO downlink with reduced feedback. Threshold policy in company with transmission mode adaptation are employed to handle feedback cost and improve system performance. Each MS carries out channel estimation, selects appropriate transmission mode and feeds back information adaptively. With feedback CSI, BS applies greedy scheduling method to activate the user of the maximum rate in each time slot. One of the proposed strategies employs single-level threshold $\left(G S_{M A} w S L T\right)$. The other one employs doublelevel threshold $\left(G S_{M A} w D T\right)$. Results show that with properly designed thresholds, both schemes can greatly reduce feedback load and achieve high throughput as well as low outage probability. Moreover, compared with $G S_{M A} w S L T, G S_{M A} w D T$ can further reduce the load and maintain the same outage performance at the cost of negligible throughput loss.

An interesting extension of this paper is to consider more generalized scenario where more users are scheduled from the pool of MS candidates. In this case, multiuser scheduling and MUI elimination should be taken into account. It can be expected that with properly 
designed thresholds the modified strategy could also result in good performance. Moreover, this paper uses numerical searching in threshold design. This method is feasible in theoretical analysis. If the relation of threshold values and system parameters (such as antenna configuration, link statistics, target outage probability, and etc.) could be specified, the proposed strategies would be of more practical significance.

\section{APPENDIX I \\ DERIVATION OF $\beta$}

The threshold for the secondary eigenmode, $Q_{\text {sec }}^{\text {th }}$ should be designed to ensure that secondary subchannel be appropriately activated, i.e. when $\lambda_{k, 1}^{2} \geq Q_{\text {pri }}^{t h}$ and $\lambda_{k, 2}^{2} \geq Q_{\text {sec }}^{t h}$ (5) should hold. $\beta$ is derived as follows,

Under $\lambda_{k, 1}^{2} \geq Q_{p r i}^{t h}$ and $\lambda_{k, 2}^{2} \geq Q_{s e c}^{t h}=\frac{\left(N_{T}-1\right) Q_{p r i}^{t h}}{1+\gamma_{0} Q_{p r i}^{t h} / N_{T}}+$ $\beta$. If (5) does not hold, i.e. $R_{k}^{S M}<R_{k}^{B F} \Leftrightarrow$ $\lambda_{k, 2}^{2}<\frac{\left(N_{T}-1\right) \lambda_{k, 1}^{2}}{1+\gamma_{0} \lambda_{k, 1}^{2} / N_{T}}$. We have

$$
\frac{\left(N_{T}-1\right) Q_{p r i}^{t h}}{1+\gamma_{0} Q_{p r i}^{t h} / N_{T}}+\beta<\frac{\left(N_{T}-1\right) \lambda_{k, 1}^{2}}{1+\gamma_{0} \lambda_{k, 1}^{2} / N_{T}}
$$

and

$$
\beta<\frac{\left(N_{T}-1\right)\left(\lambda_{k, 1}^{2}-Q_{p r i}^{t h}\right)}{\left(1+\gamma_{0} \lambda_{k, 1}^{2} / N_{T}\right)\left(1+\gamma_{0} Q_{p r i}^{t h} / N_{T}\right)}
$$

Since $\lambda_{k, 1}^{2} \geq Q_{p r i}^{t h}$, the RHS of (36) is non-negative.

Based on the analysis above, it can be readily deduced that as long as $\beta$ satisfies (37), (5) would hold under $\lambda_{k, 1}^{2} \geq Q_{p r i}^{t h}$ and $\lambda_{k, 2}^{2} \geq Q_{s e c}^{t h}$.

$$
\begin{aligned}
\beta & =\frac{N_{T}\left(\lambda_{k, 1}^{2}-Q_{p r i}^{t h}\right)}{\left(1+\gamma_{0} Q_{p r i}^{t h} / N_{T}\right)^{2}} \\
> & \frac{\left(N_{T}-1\right)\left(\lambda_{k, 1}^{2}-Q_{p r i}^{t h}\right)}{\left(1+\gamma_{0} \lambda_{k, 1}^{2} / N_{T}\right)\left(1+\gamma_{0} Q_{p r i}^{t h} / N_{T}\right)}
\end{aligned}
$$

(7) is achieved.

\section{ACKNOWLEDGMENT}

This work was supported by National Science Fund for Distinguished Young Scholars (60725105), National Basic Research Program of China (973 Program) (2009CB320404), Program for Changjiang Scholars and Innovative Research Team in University (IRT0852), the National Nature Science Foundation of China (60902032), the 111 Project (B08038), the Key Project of Chinese Ministry of Education (107103), the Fundamental Research Funds for the Central Universities (JY10000901030).

\section{REFERENCES}

[1] M. Costa, "Writing on dirty paper," IEEE Trans. on Inf. Theory, vol. 29, no.3, pp. 439-441, 1983.

[2] Lai-U Choi, R.D. Murch, "A transmit preprocessing technique for multiuser MIMO systems using a decomposition approach," IEEE Trans. on Wireless Commun., vol. 3, no. 1, pp. 20-24, 2004.

[3] T. Yoo, A.J. Goldsmith, "On the optimality of multiantenna broadcast scheduling using zero-forcing beamforming," IEEE Journal on Selected Areas in Commun., vol. 24, no. 3, pp. 528-514, 2006.

[4] X. Zhang, J. Lee, H. Liu, "Low complexity multiuser MIMO scheduling with channel decomposition," in Proc. of IEEE Intern. Conf. on Wireless Commun. and Networking, Hong Kong, pp. 2452-2456, 2007.

[5] R. Chen, A.J. Goldsmith, R.W. Heath, Z. Shen, "Lowcomplexity user and antenna selection for multiuser MIMO systems with block diagonalization," in Proc. of IEEE Intern. Conf. on Acoustics, Speech, and Sig. Process., Honolulu, pp. 613-616, 2007.

[6] W. Zhang, K.B. Letaief, "MIMO Broadcast Scheduling with Limited Feedback," Proc. of IEEE Journal on Selected Areas in Commun., vol. 25, no.7, pp. 1457-1467, 2007.

[7] W. Xu, C. Zhao, Z. Ding, "Efficient User Scheduling under Low Rate Feedback for Correlated MIMO Broadcast Channels," in IEEE Intern. Conf. on Commun., Beijing, pp. 3658-3663, 2008.

[8] D. Gesbert, M.-S. Alouini, "How much feedback is multiuser diversity really worth?" in Proc. of IEEE Intern. Conf. on Commun., Paris, pp. 234-238, 2004.

[9] S. Sanayei, A. Nosratinia, "Exploiting multiuser diversity with only 1-bit feedback," in Proc. of IEEE Wireless Commun. and Networking Conf., Sydney, pp. 978-983, 2005.

[10] P. Viswanath, D.N.C. Tse, R. Laroia, "Opportunistic beamforming using dumb antennas," IEEE Trans. on Inf. Theory, vol. 48, no.6, pp. 1277-1294, 2002.

[11] Il-Min Kim, Seung-Chul Hong, et al., "Opportunistic beamforming based on multiple weighting vectors," IEEE Trans. on Wireless Commun., vol. 4, no.6, pp. 2683-2687, 2005.

[12] M. Nicolaou, A. Doufexi, S. Armour, "Reducing feedback requirements of the multiple weight opportunistic beamforming scheme via selective multiuser diversity," in Proc. of IEEE Veh. Technol. Conf., Singapore, pp. 1-5, 2008.

[13] Z. Li, J. Yang, J. Li, J. Yao, "Opportunistic Transmission in MU-MIMO Downlink with Reduced Feedback and Mode Adaptation," in Proc. of Intern. Conf. on Wireless VITAE, Aalborg, pp. 379-383, 2009.

[14] A. Forenza, M.R. McKay, et al., "Adaptive MIMO Transmission for Exploiting the Capacity of Spatially Correlated Channels," IEEE Trans. on Veh. Technol., vol. 56, no. 2, pp. 619-629, 2007.

[15] X. Tang, M.-S. Alouini, A.J. Goldsmith, "Effect of channel estimation error on M-QAM BER performance in Rayleigh fading," IEEE Trans. on Commun., vol. 47, no. 12, pp. 18561864, 1999.

[16] J.S. Evans, "Optimal resource allocation for pilot symbol aided multiuser receivers in Rayleigh faded CDMA channels," IEEE Trans. on Commun., vol. 50, no. 8, pp. 13161325, 2002.

[17] Y. Shao, J. Yuan, "A lower bound to the sum-rate of MIMO broadcast channels with limited-rate feedback," in Proc. of IEEE Intern. Conf. on Commun., Beijing, pp. 3674-3678, 2008.

[18] D.P. Palomar, J.R. Fonollosa, "Practical Algorithms for a Family of Waterfilling Solutions," IEEE Trans. on Sig. Process., vol. 53, no. 2, pp. 686-695, 2005. 
[19] K. Zhang, Z. Niu, "Multiuser MIMO Downlink Transmission Over Time-Varying Channels," in Proc. of IEEE Intern. Conf. on Commun., Glasgow, pp. 5514-5518, 2007.

[20] A. Zanella, M. Chiani, M.Z. Win, "A General Framework for the Distribution of the Eigenvalues of Wishart Matrices," in Proc. of IEEE Intern. Conf. on Commun., Beijing, pp. 1271-1276, 2008.

Zhao Li was born in Xi'an, China. He received the B.S degree, M.S. degree and the Ph.D. degree in telecommunication engineering from Xidian University, Xi'an, China in 2003, 2006 and 2010, respectively. Since 2006, he has been with the State Key Laboratory of Integrated Service Networks at Xidian University. His research interests are in the areas of MIMO systems, multiuser communications and resource management in wireless networks.

Jiawei Yang was born in Jiangsu Province, China. He received the B.S. degree of remote sensing from Harbin Engineering University, Harbin, China in 1970, and the M.S. degree of telecommunication engineering from Xidian University, Xi' an, China in 1983. He was a visiting scholar at the University of Liverpool, Britain, from 1988 to 1990 . He is a professor of telecommunication engineering at Xidian University. His research interests focus on wireless communications, characteristics of radio propagation and data transmission. Professor Yang is the senior member of China institute communications and Chinese institute of electronics.

Junliang Yao was born in Shanxi Province, China. He received the B.S. degree in telecommunication engineering from North China Electric Power University, Hebei Province, China in 2005. He is currently working towards the Pd.D. degree in the State Key Laboratory of Integrated Service Networks at Xidian University, Xi'an, China. His research interests are in the areas of signal processing and wireless communications. 